\title{
Dynamics in the hemomicrocirculatory bed of the cattle honeycomb bag
}

\author{
Valentina Shpygova $^{1,{ }^{*}}$, Olga Dilekova $^{1}$, Viktor Mikhaylenko ${ }^{1}$, Vladimir Meshcheryakov $^{1}$, \\ and Nikolai Agarkov ${ }^{1}$
}

${ }^{1}$ Stavropol State Agrarian University, Zootekhnichesky lane, 12, Stavropol, 355017, Russia

\begin{abstract}
The study was conducted on 60 heads of black-and-white cattle of six age groups: 1 day, 30 days, 3 months, 6 months, 18 months and cows 4-5 years old. Injection, morphological and morphometric methods were used. It was found that the hemomicrocirculatory bed of the honeycomb bag has typical links. The diameter of arterioles in the firstorder ridges increases by $30.5 \%$ during the study period, but significant changes are observed only between the age groups of 3 months -6 months, 6 months- 18 months, these differences are $8.3 \%$ and $10.7 \%$, respectively. In the capillary link, significant changes are observed between the age groups of 6 months- 18 months and 18 months- $4-5$ years, which is $10.0 \%$ and $24.8 \%$, respectively. In the link of postcapillary venules, the diameter of the vessels during the study period increases by $16.9 \%$. In the link of collecting venules, significant changes $(\mathrm{p} \leq 0.01)$ in the diameter of vessels were observed between the age groups of 3-6 months and 6-18 months, which is $22.9 \%$ and $8.2 \%$, respectively. In lactating cows (4-5 years old), in addition to wide capillaries, we found postcapillary venules with a diameter of 14.80 to $27.20 \mu$ in the papillae of the lamina mucosa propria.
\end{abstract}

\section{Introduction}

The uniqueness of ruminants is in their ability to convert roughages into products with high nutritional value. The peculiarities of feed intake, digestion, distribution, role in the ecosystem, as well as the phylogenetic relationship of ruminant species do not cease to interest scientists who seek to use the new information for increasing their productivity and find ways to improve ruminants' genotype [1,2]. The variety of existing morphophysiological systems of ruminants can be considered as a pool from which some traits can be selected as targets for breeding programs for domestic ruminants $[3,4,5,6]$. The use of industrial technologies in dairy farming involves the thorough grinding of feeds, that helps to improve the efficiency of digestion, but affects the relief of the mucous membranes of the pre-ventricles, especially the honeycomb bag that performs the sorting function $[7,8,9]$. The mucosa of the adult animals' honeycomb bag forms first-order ridges and may have from four to eight facets where lower second- and third-order ridges are formed. On the bottom and in the ridges of the cells there is a large number of conical shaped papillae. To date, it is a priority opinion that the mechanism of sorting the contents of the

\footnotetext{
* Corresponding author: spygova@yandex.ru
} 
honeycomb bag is based on the physical separation of its contents, including the processes of flotation and sedimentation $[10,11]$. In the mesh, the musculature of the mucosa plays an important role in the contraction of the cell ridges. The contraction of the ridges and the honeycomb bag walls are synchronous. Synchronism in the contraction of ridges is due to the specific location of their muscles [1]. The similarity in the macroscopic anatomy of the honeycomb bag was revealed in different species of wild ruminants, which differ in the type of feeding.

Herbivores face the dilemma that the level of feed intake is negatively related to factors such as the thoroughness of grinding feed while chewing and keeping it in the digestive tract. $[11,12,13]$. In addition to the sorting function, the honeycomb bag of ruminants has the function of absorbing nutrients, but to a much less extent than the rumen [14]. The absorption of nutrients occurs in the metabolic link of the hemomicrocirculatory bed of the mucous membrane. The sources of blood supply in the multicameral stomach and the blood supply of the chambers are described in a large number of ruminants $[15,16]$, and the relationship between the microcirculatory bed of the lamina mucosa propria and the deep layers of the pre-ventricular epithelium is abundant in the papillae of the rumen, honeycomb bag and omasum [1]. The study of age-related morphometric features of the microcirculatory bed has become the aim of our research.

\section{Materials and Methods}

The material for the research was 60 heads of black-and-white cattle of six age groups: 1 day, 30 days, 3 months, 6 months, 18 months and cows 4-5 years old. The slaughter of calves aged from 1 day to 3 months was carried out for scientific and production purposes in the conditions of slaughterhouses. The slaughter of animals of older age groups was carried out in order to obtain livestock products. All manipulations on selection of experimental material were carried out according the Directive 2010/63/EU of the European Parliament and the Council of the European Union 2010/09/22 "On Protection of Animals Used for Scientific Purposes".

To detect the submucosal plexus of the blood bed of the honeycomb bag, the vessels were injected with a contrast suspension prepared according to the prescription: glycerin $100 \mathrm{ml}$, barium sulfate (BaSO4) - $100 \mathrm{~g}$, aqua destillata - 400-500 ml. The contrast suspension was injected at a pressure of $110-120 \mathrm{~mm}$ through a device with a pressure gauge. For injection into the veins, a white suspension was used, and before pouring into the arteries, a red pigment was added to it. After settling of the suspension particles and its solidification, the organ (mesh) was subjected to stratification into the mucosal and submucosal, muscular and serous layers. Histological sections were prepared in the classical way, stained with hematoxylin-eosin, iron hemnoxylin-picrofuxin according to Van Gieson, resorcinol-fuchsin according to Weigert, phosphor-tungsten hematoxylin according to Mallory. Histological preparations were examined using an OLYMPUS-BX 43 light microscope (Japan) and an OLYMPUS C 300 camera (Japan). Vascular morphometry was performed in the program "Video Test - master Morphology 4.0" for Windows (Russia).

\section{Results}

The data obtained during the study of the hemomicrocirculatory bed of the honeycomb bag wall indicated its typical structure and age-related features.

Intrahepatic arteries of the muscular type, which supply blood to the mesh, passing into the submucosal layer, successively give branches to the serous membrane. There they form 
the subsurface plexus. Then, in the submucosal layer an arterial plexus is formed, which supplies blood to the muscle and mucous membranes. The hemomicrocirculatory bed of the serous membrane represents repeating geometric figure of polygonal shape with a diameter of arterioles from 20 to $40 \mu$, where precapillary arterioles, or metarterioles, depart. The collateral arteriole often passes with an arteriole of a larger caliber. In this paper we do not provide complete data on the morphometry of the vessels of the microcirculatory bed of the serous membrane, but it should be noted that its main age characteristic is an increase in the density of the capillary link per square unit of area, thus, in adult animals, the capillary link of the module prevails.

From the submucosal plexus, arterial vessels of various calibers are directed to the muscle membrane and form an intermuscular plexus in it, where all five links of the hemomicrocirculatory bed can be distinguished (fig. 1).

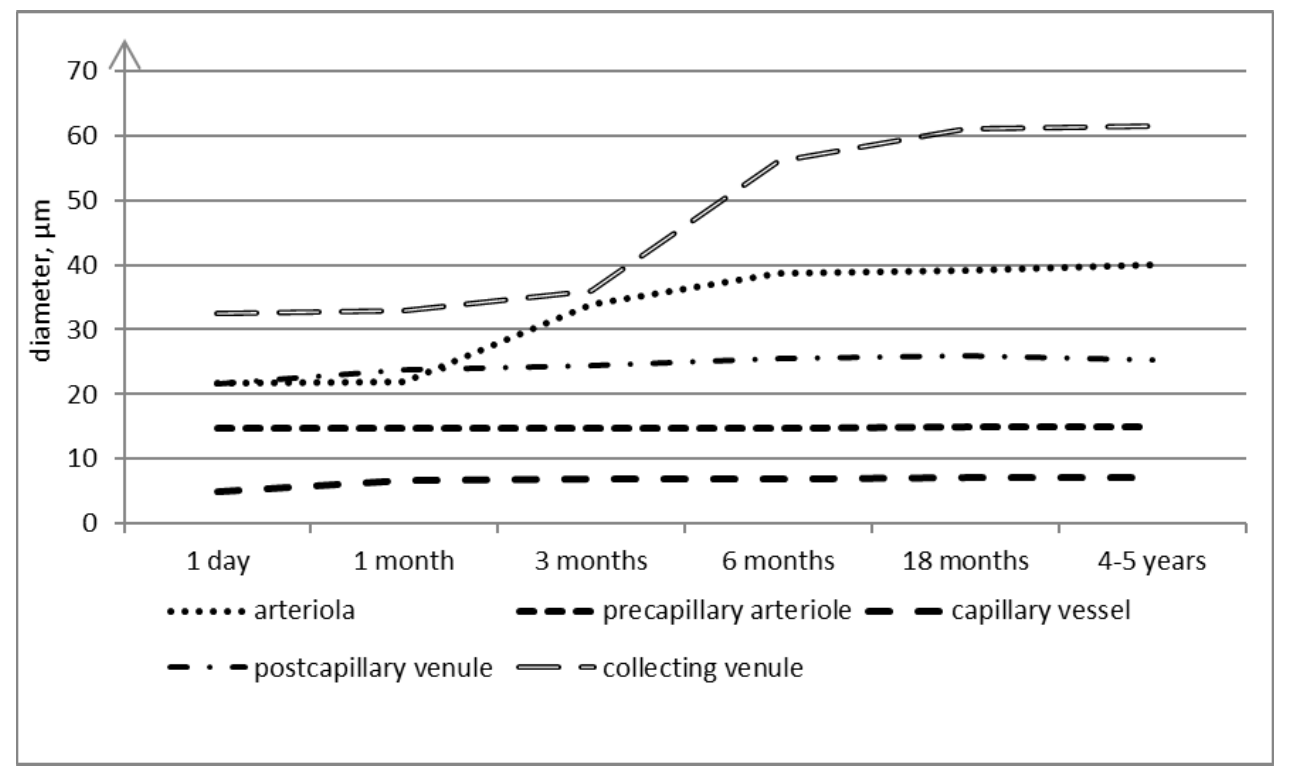

Fig. 1. Dynamics in the vessels diameters of the hemomicrocirculatory bed of the honeycomb bag muscular membrane.

An increase in the number of arterioles with a large diameter is observed in the groups of calves of three-month and six-month age. The average diameter of arterioles significantly $(\mathrm{p}<0.01$ ) increases by $54.3 \%$ and $12.3 \%$ respectively, compared to the previous age groups of calves. In older age groups, there is a statistical stability in this parameter. During the study period, there were no significant changes in the link of precapillary arterioles. Their diameters within the variation series have a minimum maximum value from 12.3 to $18.7 \mu$. In the capillary link, there are significant changes $(p \leq 0.01)$ only between groups of calves aged 1 day and 1 month, that is $34.1 \%$. However, in adult animals (4-5 years old) compared to newborns, the diameter of the capillaries is $43,0 \%$ larger. The minimum diameter is noted in newborns of $3.6 \mu$, the maximum in cows of $4-5$ years $-8.7 \mu$, with average statistical values of $5.02 \pm 0.15 \mu$ and $7.18 \pm 0.17 \mu$, respectively. In the link of postcapillary venules, significant changes $(p \leq 0.01)$ were also observed only between groups of calves aged 1 day and 1 month, which is $9.5 \%$. However, over the entire study period, this morphometric parameter increases by $16.9 \%$. In the link of collecting venules, significant differences $(\mathrm{p} \leq 0.01)$ were observed between the age groups of 1 month -3 months, 3 months -6 months and 6 months- 18 months. These differences are $9.4 \%, 55.2 \%$ and $10.8 \%$ respectively. 
In the submucosal plexus organ artery branched to the third order at the age of 1 month, to the fourth order at the age of 3 months, up to the fifth order at the age of 6 months, up to the sixth order at the age of eighteen months and older. The vessels form polygonal networks, oriented mainly along the base of the cells. Vessels detected by contrast suspension filling have a diameter of up to $50 \mu$ (fig. 2).
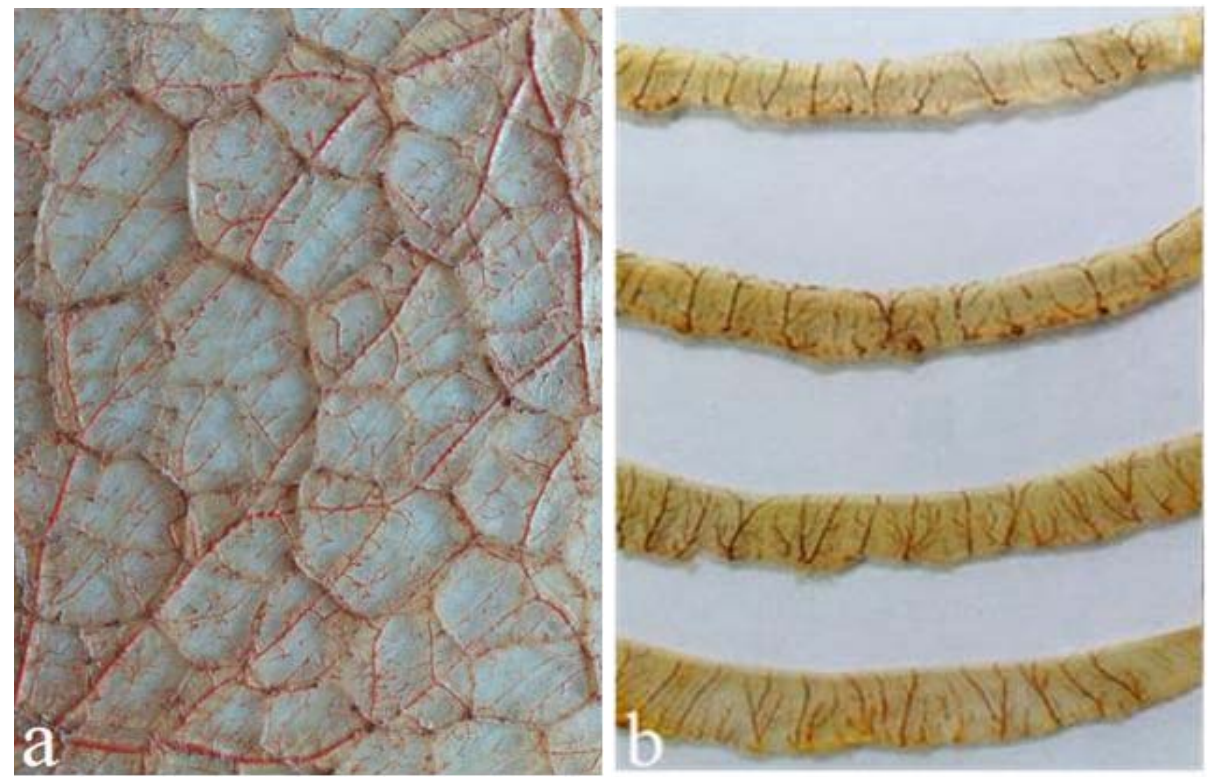

Fig. 2. a) submucosal arterial plexus of the honeycomb bag; b) arterial vessels with different levels of division in the ridges of the first-order reticulum cells, male, age 6 months.

In newborns and animals aged 1 month, at the base of the first-order cells ridges, there are muscle-type arteries with a diameter of 100 to $150 \mu$. In newborns at the age of 1 day, the second-order ridges are nourished from arterioles with a diameter of 48 to $100 \mu$ that pass parallel to each other, across the ridge of the cells at their base. The vessels form polygonal networks, oriented mainly along the base of the cells. Vessels detected by contrast suspension filling have a diameter of up to $50 \mu$. In animals aged six to eighteen months and older, muscle-type arteries with a diameter of 300 to $600 \mu$ pass at the base of the first-order cells ridges. Arterioles of various calibers are directed into the mucosa of the honeycomb bag from the vessels of the submucosal plexus, which are divided into capillaries in their lamina mucosa propria. In newborn animals, the papillae include 1-2 papillary arterioles, in adult animals there are 5-7 of them. During the study period, the diameter of arterioles of the first-order ridges increases by $30.5 \%$, but significant changes are observed only between the age groups of 3 months -6 months, 6 months- 18 months, and these differences are $8.3 \%$ and $10.7 \%$, respectively (fig. 3). Arterioles give off precapillary arterioles, or metarterioles, along the entire length, which then disintegrate into metarterioles and capillaries. The arteriole itself also passes into the metarteriole. Due to the many anastomoses of arterioles with muscle-type arteries, metarterioles, and capillaries, the conditions for blood delivery to the capillary pool are created, and the disruption of the functioning of each vessel can be compensated by the work of neighboring arterioles due to the redistribution of blood through the anastomoses. There were no significant changes in the diameter of the precapillary arterioles between the age groups, but during the study period, the average values of this parameter increased by $11.7 \%$. Precapillary arterioles are divided in the lamina mucosa propria of the honeycomb bag ridges cells of various levels, the cone-shaped and spherical papillae of the bottom and in 
the interstitial spaces to capillaries with a diameter of $6.56 \pm 2.78 \mu$ in newborn calves of one day age and a diameter of $7.70 \pm 1.29 \mu$ in adult animals of $4-5$ years old.

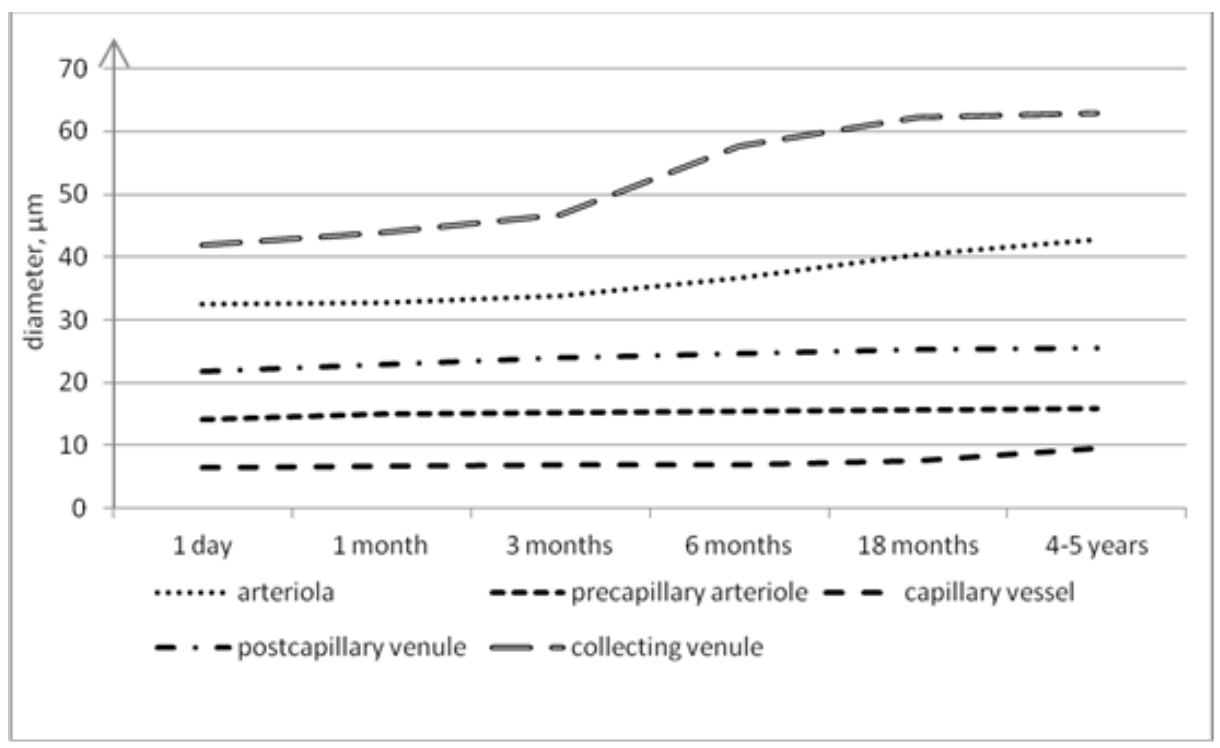

Fig. 3. Dynamics in vessel diameters of the hemomicrocirculatory bed of the mucous membrane in the honeycomb bag.

In the capillary link, significant changes were observed between the age groups of 6 months -18 months and 18 months $-4-5$ years, that is $10.0 \%$ and $24.8 \%$, respectively (fig. 4). Animals of 4-5 years old were lactating cows.

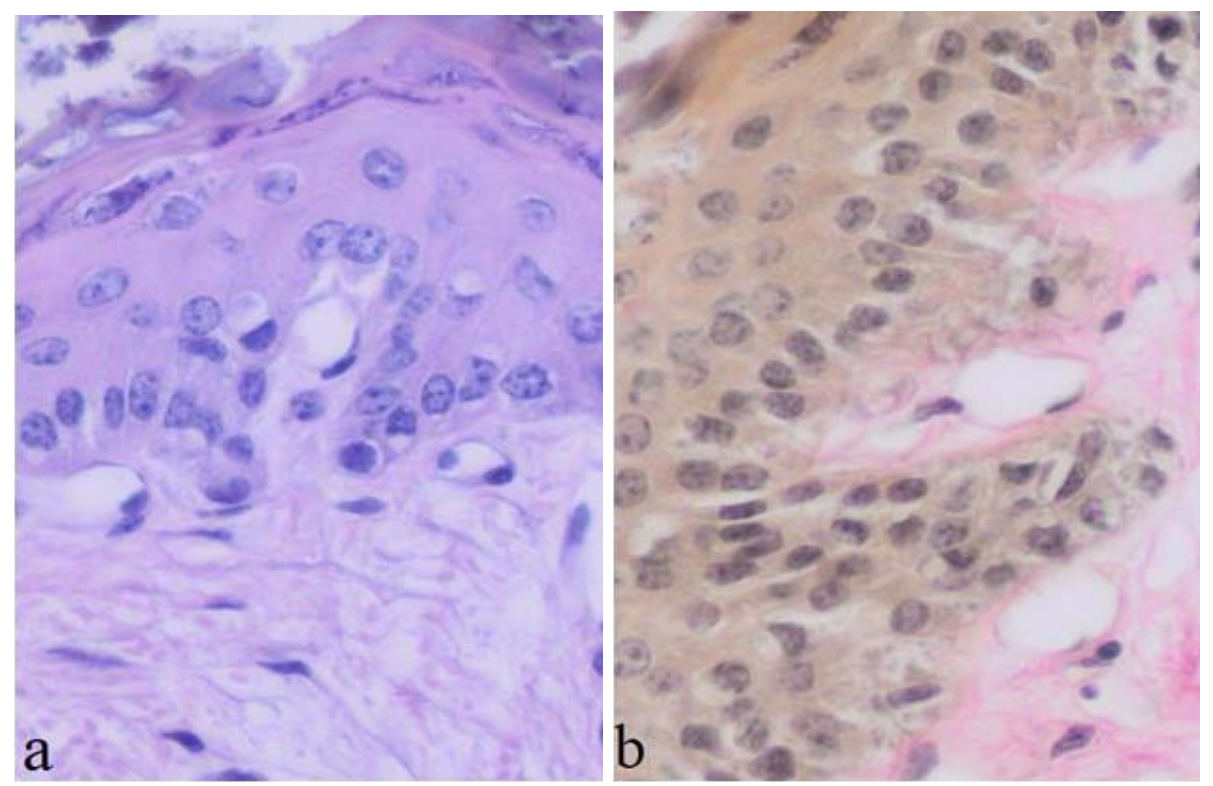

Fig. 4. Capillaries in the papillae of the lamina mucosa propria in the first-order cells' plica, where: a) Staining with hematoxylin and eosin; b) staining by Van Gieson; cow, age 4 years. Zoom $\times 400$.

In the link of postcapillary venules, the vessel diameters do not significantly differ between age groups, but during the study period this morphometric parameter increases by 
$16.9 \%$. Total number of vessels per unit area increases as well. In the link of collecting venules, significant changes $(\mathrm{p} \leq 0.01)$ in the diameter of vessels were observed between the age groups of 3-6 months and 6-18 months, that is $22.9 \%$ and $8.2 \%$, respectively.

\section{Conclusions}

The hemomicrocirculatory bed of the honeycomb bag has typical links: arterioles, precapillaries, capillaries, postcapillaries, collecting venules. Morphofunctional changes in each layer of the honeycomb bag take place most significantly during the period of intensive growth of epithelial connective tissue formations (ridges) of the honeycomb bag mucosa, so the dynamics of morphometric parameters of the hemomicrocirculatory bed in this mucous membrane is most noticeable. During the study period, the diameter of arterioles in the first-order ridges increases by $30.5 \%$, but significant changes are observed only between the age groups of 3 months -6 months, 6 months- 18 months, these differences are $8.3 \%$ and $10.7 \%$, respectively. In animals of different age groups, there are no significant changes in the diameter of precapillary arterioles, but during the study period, the average values of this parameter in cows of 4-5 years old are $11.7 \%$ higher than in newborn calves. In the capillary link, significant changes are observed between the age groups of 6 months- 18 months and 18 months- $4-5$ years, that are $10.0 \%$ and $24.8 \%$, respectively. In the link of postcapillary venules, the vessel diameters between the age groups did not significantly differ, but during the study period, this morphometric parameter increased by $16.9 \%$. In the link of collecting venules, significant changes $(p \leq 0.01)$ in the diameter of vessels were observed between the age groups of 3-6 months and $6-18$ months, which is $22.9 \%$ and $8.2 \%$, respectively. Cattle honeycomb bag performs the function of absorbing nutrients in a smaller volume than the rumen. Perhaps this explains the fact that in the papillae of the lamina mucosa propria in lactating cows, besides wide capillaries, postcapillary venules with a diameter of 14.80 to $27.20 \mu$ have been found. Subepithelialally, postcapillaries are formed in the mucosa when several capillaries merge, as well as 2-4 collecting venules in young animals and 4-10 or more collecting venules in adult animals.

\section{References}

1. W. Perez, H.E. Koenig, H. Jerbi et al., Vertebrate zoology 66(3), 419-425 (2016)

2. M. Clauss, R.R. Hofmann, Ecology, evolution and behaviour of wild cattle: implications for conservation (2014) DOI: https://doi.org/10.1017/CBO9781139568098.008

3. K.H. Baker, H.W.I. Gray, V. Ramovs et al., Heredity 119(1), 16-26 (2017) DOI: 10.1038/hdy.2017.11

4. M. Clauss, J. Fritz, A. Tschuor et al., Journal of animal physiology and animal nutrition 101(1), 61-69 (2017) DOI: 10.1111/jpn.12505

5. J. Wang, H. Li, L. Zhang, Y. Zhang, M. Yue, B. Shao, J. Wang, Int. J. Morphol. 32(3), 871-881 (2014) DOI: http://dx.doi.org/10.4067/S0717-95022014000300021

6. W. Pérez, S. Erdogan, R. Ungerfeld, Anat. Histol. Embryol 44(1), 43-49 (2015) DOI: 10.1111/ahe.12106

7. Franco, J. Masot, A. García, E. Redondo, Anat. Histol. Embryol 41(5), 362-73 (2012) DOI: $10.1111 / \mathrm{j} .1439-0264.2012 .01146 . \mathrm{x}$

8. H. Jerbi, M. Bayoudh, M. Clauss, W. Pérez, Int. J. Morphol. 34(4), 1266-1270 (2016) http://dx.doi.org/10.1590/S1806-92902017000700008 
9. E. Redondo, A. Garcia, C. Ortega, F.J. Pena, A. Gazquez, J. Masot, Animal science journal 91(1), e13319 (2020) DOI: 10.1111/asj.13319

10. A. Garcia, P. Rodriguez, J. Masot, A. Franco, E. Redondo, Animal Science Journal 85(11), 951-962 (2014) https://doi.org/10.1111/asj.12231

11. M. Clauss, J. Hummel, Revista Brasileira de Zootecnia 46(7), 606-613 (2017) http://dx.doi.org/10.1590/S1806-92902017000700008

12. P. Hejcmanová, S. Ortmann, L. Stoklasová, M. Clauss, Comparative Biochemistry and Physiology Part A: Molecular \& Integrative Physiology 246, 110720 (2020) https://doi.org/10.1016/j.cbpa.2020.110720

13. J. Hummel, S. Hammer, C. Hammer, J. Ruf, M. Lechenne, M. Clauss, Comp. Biochem. Physiol. A 182, 22-26 (2015) https://doi.org/10.1016/j.cbpa.2014.12.006

14. R. Rackwitz, F. Dengler, G. Gabel, Animals 10(12), 2198 (2020) DOI: 10.3390/ani10122198

15. W. Pérez, N. Vazquez, R. Ungerfeld, Anat. Histol. Embryol 45(3), 240-245 (2016) DOI: $10.1111 /$ ahe. 12192

16. V.M. Shpygova, O.V. Dilekova, V.V. Mikhaylenko, V.A. Meshcheryakov, N.A. Pisarenko, Research Journal of Pharmaceutical, Biological and Chemical Sciences 9(6), 1234-1238 (2018) 